\title{
Diversite Floristique des Plantes à Potentialité Décorative Issues des Formations Naturelles du Nord de la Côte d'Ivoire
}

\author{
Kwassi Dauphner Serges S. Amani, \\ Laboratoire de Botanique, Université Félix Houphouët-Boigny, \\ Côte d'Ivoire, Abidjan \\ Faustine Akossoua Kouassi, \\ Centre National de Floristique (CNF)
}

Emma Ablan Aké-Assi, Laboratoire de Botanique, Centre National de Floristique (CNF), Université Félix Houphouët-Boigny, Côte d'Ivoire, Abidjan; Institut Botanique AkéAssi d'Andokoi (IBAAN), Abidjan, Côte d'Ivoire

Doi: 10.19044/esj.2019.v15n15p46 URL:http://dx.doi.org/10.19044/esj.2019.v15n15p46

\section{Résumé}

Cette étude réalisée dans le domaine soudanais de la Côte d'Ivoire a pour objectif de contribuer à la sauvegarde de la flore ivoirienne à travers la valorisation des plantes à potentialité décorative du Nord de la Côte d'Ivoire. La méthode d'inventaire utilisée est le relevé itinérant. Elle a consisté à parcourir dans différentes directions autour des grandes villes ayant différents milieux et à recenser toutes les espèces végétales à potentialité décorative rencontrées, sans tenir compte de leur taille. Ces espèces ont été analysées au Centre National de Floristique (CNF). Ces relevés nous ont permis de récolter 287 espèces végétales réparties entre 220 genres et 79 familles botaniques, avec une prédominance des herbes. Les familles les plus abondantes sont celles des Fabaceae, des Apocynaceae et des Acanthaceae. Parmi ces espèces, $73,5 \%$ sont des Phanérophytes et $0,3 \%$ des épiphytes. Aussi, une espèce endémique ivoirienne (GCI) Cyphostemma ornatum A.Chev. exHutch. \& Dalziel (Vitaceae) a été également recensée. Treize espèces soit 4,52\% sont considérées comme espèces introduites (i). Au niveau écologique, des espèces hydrophytes et l'espèce comme Senna podocarpus Guill. \&Perr. (Fabaceae) qui est une des plantes rustiques a été inventoriée. Ces résultats montrent une grande richesse floristique décorative dans le domaine soudanien. Ces travaux ont contribué à la connaissance de la composition et de la diversité des plantes à potentialité décorative du domaine soudanais de la Côte d'Ivoire. 
Mots-clés : Diversité, Plante ornementale, domaine soudanais, Côte d'Ivoire

\title{
Floristic Diversity of Plants with Decorative Potentiality from Natural Training in the Northern Cote d'Ivoire
}

\author{
Kwassi Dauphner Serges S. Amani, \\ Laboratoire de Botanique, Université Félix Houphouët-Boigny, \\ Côte d'Ivoire, Abidjan
}

Faustine Akossoua Kouassi, Centre National de Floristique (CNF)

Emma Ablan Aké-Assi,

Laboratoire de Botanique, Centre National de Floristique (CNF), Université Félix Houphouët-Boigny, Côte d'Ivoire, Abidjan; Institut Botanique AkéAssi d'Andokoi (IBAAN), Abidjan, Côte d'Ivoire

\begin{abstract}
This study carried out in the Sudanese field of Côte d'Ivoire aims to contribute to the safeguarding of Ivorian flora through the valorization of plants with decorative potentiality of the North of Côte d'Ivoire. The inventory method used is the roaming survey. It consisted of walking in different directions around large cities with different backgrounds and listing all the plant species with decorative potential encountered, regardless of their size. These species have been analyzed at the National Center for Floristics (NCF). These surveys allowed us to collect 287 plant species divided between 220 genera and 79 botanical families, with a predominance of herbs. The most abundant families are Fabaceae, Apocynaceae and Acanthaceae. Of these species, $73.5 \%$ are Phanerophytes and $0.3 \%$ epiphytes. Also, an Ivorian endemic species (GCI) Cyphostemma ornatum A.Chev. exHutch. \& Dalziel (Vitaceae) was also enumerated. Thirteen species or $4.52 \%$ are considered introduced species (i). At the ecological level, hydrophytic species and the species like Senna podocarpus Guill. \& Perr. (Fabaceae) which is a hardy plant has been inventoried. These results show a great decorative floristic richness in the Sudanian field. This work contributed to the knowledge of the composition and the diversity of the plants with decorative potentiality of the Sudanese field of Côte d'Ivoire.
\end{abstract}


Keywords: Diversity, Ornamental plant, Sudanese domain, Ivory Coast

\section{Introduction}

La dégradation du couvert végétal en Afrique est en grande partie due à l'agriculture, à la croissance démographique et aux facteurs climatiques (Ehui et al., 1989 ; Wezel et al., 2000 ; Bamba et al., 2010). En Côte d'Ivoire, les surfaces forestières qui se chiffraient à 15 millions d'hectares dans les années 1900 (Sodefor, 1996), sont aujourd'hui estimées à moins de 4 millions d'hectares (Bnetd, 2015). Cette régression du couvert végétal est liée aux activités anthropiques (exploitation forestière abusive, agriculture extensive etc.) d'une population en perpétuelle croissance (Kouamé, 1998). Dans le nord du pays, en zone soudanienne, les forêts claires, sèches et les savanes n'ont pas aussi été épargnées, car elles ont également fourni des devises à travers la culture du coton et de l'anacarde (Djato, 2001 ; Sangne, 2009). Ces cultures ont ainsi contribué à la disparition ou la raréfaction d'un nombre important d'espèces végétales, dont celles à potentialité décorative abritées par ces écosystèmes. Cette diminution des ressources a aussi eu un impact sur les services écosystémiques que procure la biodiversité aux populations locales, notamment les services de prélèvement pour l'alimentation, l'énergie, la construction, la médecine, l'artisanat (Wezel et Lykke, 2006 ; Traoré et al., 2011) et l'ornement.

Ainsi, dans un souci de conserver et améliorer la qualité de ces plantes sauvages décoratives, puis accroître leur rendement, l'homme a très tôt tenté de les apprivoiser tout en les domestiquant sur des étendues plus ou moins grandes (Mboh, 2001). Mais, au-delà de cette notion utilitaire, de nombreux peuples aiment les plantes décoratives pour leur seule beauté (Barloy, 1984). Ces plantes sont aussi aimées pour le parfum qu'exhalent certains de leurs organes et sont sollicitées pour diverses manifestations (naissances, anniversaires, mariages, obsèques, etc.). Les plantes ornementales agrémentent le cadre de vie et la fleur accompagne tous les moments d'émotion (Aké Assi et al., 2010 ; Radji et al., 2010).

$\mathrm{Vu}$ l'importance de ces plantes dans la vie quotidienne, il devient urgent de faire un état des lieux de la diversité de la flore à potentialité décorative, de la végétation et des services écosystémiques (Aké Assi, 1984 ; Yao, 2013). Très peu d'études se sont intéressées à la diversité floristique savanicole du nord et particulièrement celle de la zone Nord de la Côte d'Ivoire (Devineau et al., 1984 ; Ambé, 2001). La présente étude s'est donc donnée pour objectif général de contribuer à la sauvegarde de la flore ivoirienne à travers la valorisation des plantes à potentialité décorative du Nord de la Côte d'Ivoire. 


\section{Matériel et méthodes}

\section{Site d'étude}

L'étude s'est déroulée dans la partie nord de la Côte d'Ivoire, précisément dans le domaine soudanais. Il est localisé entre la latitude $7^{\circ} 2^{\prime}$ et $10^{\circ} 2^{\prime}$ 'Nord et la longitude $2^{\circ} 1^{\prime}$ et $8^{\circ} 2^{\prime}$ Ouest. Ce domaine est subdivisé en deux secteurs (le secteur subsoudanais et le secteur soudanais) tels que définis par Guillaumet (1967).

La végétation est composée de forêts claires, forêt galerie et de savanes. Le climat, de type soudanais est caractérisé par deux saisons dont une pluvieuse (4 à 5 mois) et une sèche qui dure de 7 à 8 mois (Bertauld, 1986). Les précipitations annuelles surviennent en majeure partie pendant les mois de juillet, août et septembre.

L'inventaire floristique s'est déroulé dans six grandes villes de la zone d'étude. Il s'agit d'Odienné, Korhogo, Ferkessédougou dans le (secteur subsoudanais) et Tingrela, Ouangolodougou, Bouna dans le (secteur soudanais). Le choix de ces villes est lié à leur superficie, aux types de formation végétale et au fait qu'aucune étude portant sur les plantes à potentialité décorative n'y a été encore réalisée.

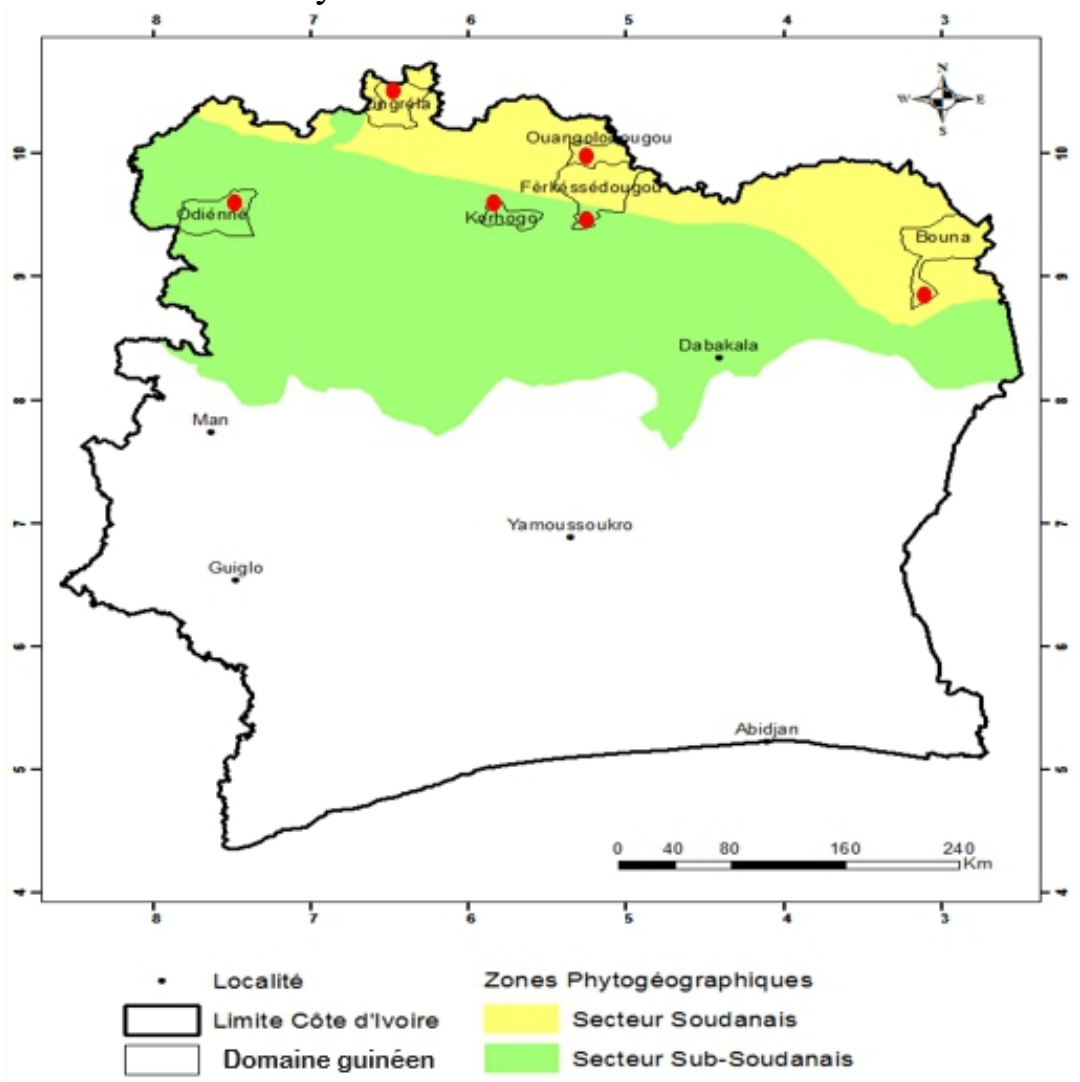

Figure 1: Carte de répartition des sites d'étude

Sources: (Guillaumet et Adjanohoun, 1971; modifié par Amani) 


\section{Collecte de données}

La collecte des données est faite par la méthode d'inventaire itinérant utilisée par plusieurs chercheurs dont Malan et al. (2007) ; Vroh (2013) ; Aké Assi E. (2015). Le relevé a consisté à recenser dans les différents milieux rencontrés, toutes les espèces à belles fleurs, à feuillage décoratif, à allure ou port décoratif. Nous avons aussi recensé les plantes odoriférantes, les plantes décoratives par les fruits et par les graines. Dans chaque secteur, les ilots de forêts et de savanes présents aux alentours des principales villes ont été choisis pour mener à bien la présente étude. Grace à l'herbier et à des ouvrages spécialisés du Centre National de Floristique (C.N.F), les espèces recensées ont été identifiées. C'est à la suite de cela que la liste floristique des espèces à potentialité décorative de l'étude a été établie.

\section{Analyse des données}

\section{Diversité qualitative de la flore ornementale}

Après la collecte des données, basée sur la beauté des organes, plusieurs analyses ont été faites. En effet, les indices de diversité ont été calculés. Le nombre d'espèces, de genres et de familles de toutes les espèces végétales rencontrées lors des inventaires a été déterminé pour chaque milieu et pour chaque secteur. Par la suite, les types biologiques et morphologiques ont été renseignés en se référant à Raunkiaer (1934) et Aké Assi (2001 ; 2002). Il en a été de même pour la répartition phytogéographique en utilisant les grandes subdivisions phytogéographiques de White (1983) et le catalogue des plantes vasculaires de la Côte d'Ivoire (Aké Assi, 2001 ; 2002). Aussi, la diversité de la flore globale a été estimée par comparaison de la présente liste à celles de Aké Assi (1984) et de l'UICN (2018). Cela a permis de mettre en évidence la présence d'espèces à statut particulier : espèces endémiques, rares et menacées d'extinction.

\section{Diversité quantitative de la flore décorative}

Pour l'analyse de la diversité quantitative de la flore ornementale des deux milieux (secteurs subsoudanais et soudanais), différents coefficients ont été calculés :

L'indice de diversité (Aké Assi, 1984), étant le rapport entre le nombre d'individus et le nombre d'espèces d'une population, a permis d'apprécier la diversité spécifique quantitative des milieux selon la formule suivante :

$$
\mathrm{Id}=\frac{\boldsymbol{N b i}}{\boldsymbol{N b} \cdot S p}
$$

Avec, Id : Indice de diversité, Nbi : Nombre d'individus, Nb.Sp. : Nombre d'espèces. 
Une flore est diversifiée lorsque les indices de familles composant cette flore sont en général faibles. Les fortes valeurs de ce coefficient caractérisent les flores peu diversifiées. Dans cette étude, nous nous sommes intéressés aux indices de diversité générique (IDg) et de famille (IDf) selon les formules suivantes: $\operatorname{IDg}=\frac{G}{E}$ avec $\mathrm{G}$ : nombre de genres, $\mathrm{E}:$ nombre d'espèces ;

$\mathrm{IDf}=\frac{E}{F}$ avec $\mathrm{F}:$ nombre de familles, $\mathrm{E}:$ nombre d'espèces.

Le coefficient de similitude selon Sorensen (1948) a été calculé suivant la formule :

$$
\text { Cs }(s)=100[2 c /(a+b)]
$$

$(\mathrm{a}=$ nombre d'espèces du milieu $\mathrm{A}, \mathrm{b}=$ nombre d'espèces du milieu $\mathrm{B}, \mathrm{c}=$ nombre d'espèces communes aux deux milieux écologiques).

Cet indice a été utilisé pour apprécier le pourcentage d'espèces communes à deux milieux comparées. Plus les deux listes floristiques comparées ont des espèces en commun, plus leur coefficient tend vers 100 .

Les analyses ont été effectuées à l'aide du logiciel XLSTAT version 2018 pour le calcul des différents indices utilisés. La classification phylogénétique, selon les travaux de l'Angiosperms Phylogeny Group APG VI (2016) a été adoptée pour nommer les espèces répertoriées.

\section{Résultats}

\section{Richesse floristique}

L'inventaire floristique de la zone d'étude a permis de recenser 287 espèces à potentialité décorative parmi lesquelles 247 sont issues du secteur subsoudanais et 68 du secteur soudanais. Ces espèces se répartissent en 220 genres appartenant à 79 familles. Les familles les mieux représentées sont les Fabaceae suivies des Apocynaceae, Acanthaceae, Asteraceae et Anacardiaceae (Tableau I).

Tableau I : Liste des familles les mieux représentées en terme d'espèces

\begin{tabular}{clcc}
\hline $\mathrm{N}^{\circ}$ & Familles & Nombre d'espèces & Proportion $(\%)$ \\
\hline 1 & Fabaceae & 34 & 11,84 \\
2 & Apocynaceae & 31 & 10,80 \\
3 & Acanthaceae & 20 & 6,96 \\
4 & Asteraceae & 20 & 6,96 \\
5 & Anacardiaceae & 16 & 5,57 \\
6 & Combretaceae & 13 & 4,52 \\
7 & Lamiaceae & 12 & 4,18 \\
8 & Euphorbiaceae & 9 & 3,13 \\
9 & Malvaceae & 9 & 3,13 \\
10 & Rubiaceae & 8 & 2,78 \\
\hline
\end{tabular}


Le secteur subsoudanais est le plus riche avec 48 familles soit $60,75 \%$ des familles recensées. Dans ce secteur, les Fabaceae sont les plus dominantes et contiennent 29 espèces soit $10,13 \%$ des espèces rencontrées dans l'ensemble de la zone. Dans le secteur soudanais, c'est plutôt les Anarcadiaceae avec 7 espèces qui sont majoritaires, soit 2,42\% des espèces de la zone totale. Au niveau générique, le nombre de genres varie d'une famille à une autre. Ainsi, deux genres sont les mieux représentés. Ce sont les genres Combretum et Lannea représentés chacun par 5 espèces soit $1,74 \%$ de l'ensemble des espèces. Ces deux genres sont rencontrés aussi bien dans le secteur subsoudanais que dans le secteur soudanais. Parmi les espèces recensées, 26 sont communes aux secteurs soudanais et subsoudanais (Tableau II).

Tableau II : Liste des espèces à potentialité décorative communes aux secteurs soudanais et subsoudanais

\begin{tabular}{|c|c|c|}
\hline $\mathbf{N}^{\circ}$ & Espèces & Familles \\
\hline 1 & Anogeissus leiocarpus Hutch & Combretaceae \\
\hline 2 & Antidesma venosum Tul. & Phyllanthaceae \\
\hline 3 & Carissa edulis Vahl & Apocynaceae \\
\hline 4 & Cochlospermum planchonii Hook.f & Bixaceae \\
\hline 5 & Combretum collinum Fresen & Combretaceae \\
\hline 6 & Combretum nigricans Lepr & Combretaceae \\
\hline 7 & Cordia myxa $\mathrm{L}$. & Boraginaceae \\
\hline 8 & Cyperus margaritaceus Vahl & Cyperaceae \\
\hline 9 & Fimbristylis ferruginea Vahl. & Cyperaceae \\
\hline 10 & Glinus lotoides L. & Molluginaceae \\
\hline 11 & Justicia insularis Anderson & Acanthaceae \\
\hline 12 & Landolphia dulcis Pichon & Apocynaceae \\
\hline 13 & Lannea acida Rich & Anacardiaceae \\
\hline 14 & Ludwigia abyssinica A. Rich & Onagraceae \\
\hline 15 & Neohyptis paniculata Baker & Lamiaceae \\
\hline 16 & Ozoroa insignis Kuntze & Anacardiaceae \\
\hline 17 & Parinari curatellifolia Planch & Chrysobalanaceae \\
\hline 18 & Phaulopsis barteri Anderson & Acanthaceae \\
\hline 19 & Pleiotaxis chlorolepis C.Jeffrey & Araceae \\
\hline 20 & Polygonum lanigerum $\mathrm{L}$. & Polygonaceae \\
\hline 21 & Saba comorensis Pichon & Apocynaceae \\
\hline 22 & Sorindeia warneckei Engl & Anacardiaceae \\
\hline 23 & Terminalia albida Scott-Elliot & Combretaceae \\
\hline 24 & Vernonia amygdalina Del & Asteraceae \\
\hline 25 & Vigna racemosa Hutch. & Fabaceae \\
\hline 26 & Vitex domengensis $\mathrm{L}$ & Lamiaceae \\
\hline
\end{tabular}

Une plante endémique ivoirienne (GCI), a été recensée. C'est l'espèce : Cyphostemma ornatum (Vitaceae) soit $0,30 \%$ des espèces. Les espèces endémiques Ouest africaines (GCW) au nombre de 3 soit $1 \%$, sont toutes localisées dans le secteur subsoudanais. 
Les taxons à potentialité décorative du domaine soudanais sont classés en 5 types morphologiques. Les plus nombreux sont les plantes herbacées représentées par 131 espèces soit 45,64\% suivies des arbustes, 76 espèces soit $26,48 \%$, des lianes 63 espèces soit 21,95\%, des arbres 16 espèces soit 5,57\%, et un Suffrutex soit $0,34 \%$. Les fortes proportions des types morphologiques n'ont été observées qu'au niveau du secteur subsoudanais (Figure 2).

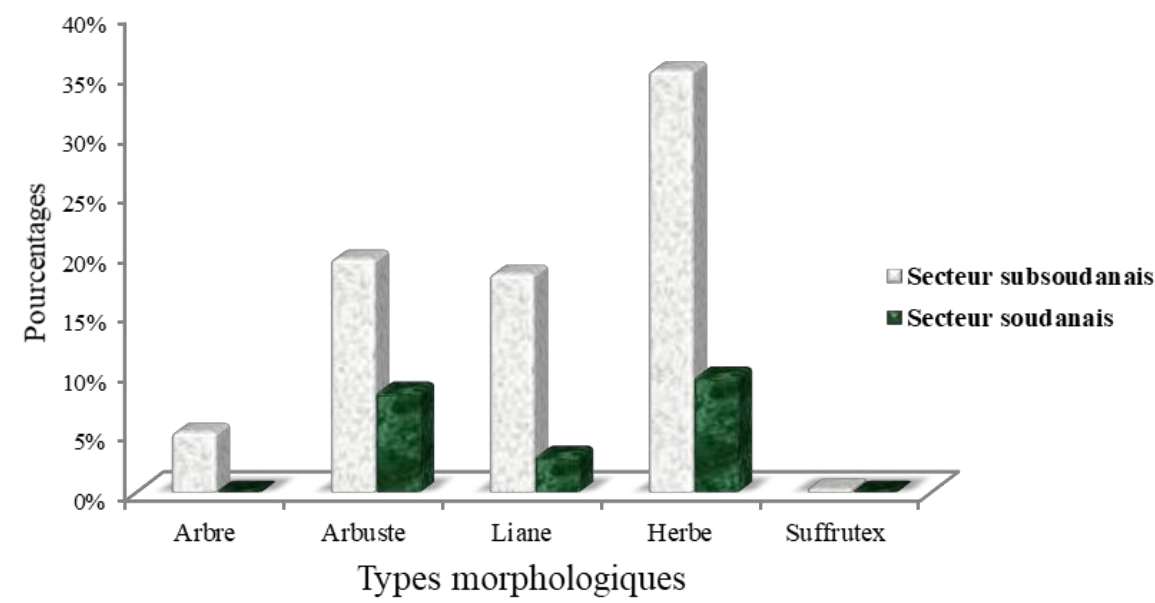

Figure 2: Répartition des plantes à potentialité ornementales, selon les types morphologiques.

La flore inventoriée comprend 11 types biologiques. L'analyse des deux secteurs montre que les Thérophytes (Th) 10\% et les Phanérophytes sont majoritaires avec 211 espèces soit (73,51\%). Parmi les Phanérophytes, on note les microphanérophytes représentées par 123 espèces soit $42,85 \%$ suivies des nanophanérophytes (np) 23,34\%.

Les microphanérophytes décoratives sont majoritairement représentés dans le domaine soudanais précisément dans les formations naturelles sacrées. Dans le secteur subsoudanais, c'est plutôt dans les savanes arbustives et quelques îlots de forêts que les microphanérophytes ont été rencontrés. Les proportions des autres types biologiques sont représentées dans le Tableau III. 
Tableau III: Répartition des plantes à potentialité ornementale, selon les types biologiques.

\begin{tabular}{cc}
\hline Types biologiques & Proportions (\%) \\
\hline $\mathrm{mp}$ & 42,9 \\
$\mathrm{np}$ & 24,0 \\
$\mathrm{Th}$ & 10,1 \\
$\mathrm{mP}$ & 6,6 \\
$\mathrm{G}$ & 5,6 \\
$\mathrm{H}$ & 4,2 \\
$\mathrm{Ch}$ & 3,5 \\
$\mathrm{Hpy}$ & 1,4 \\
$\mathrm{Hyd}$ & 0,7 \\
$\mathrm{Rh}$ & 0,3 \\
Par & 0,3 \\
Ep & 0,3 \\
\hline Total & 100
\end{tabular}

Chaméphytes :Ch ; Epiphytes :Ep ; Hémicryptophytes H ; Hémicryptophytepyrophytique:Hpy ; Hydrophytes :Hyd ; Géophytes :G ; mésophanérophyte :mP ; microphanérophyte :mp ; nanophérophyte :np ;Parasites :Par ; rhéophytes : rh ; Thérophyte : Th.

Au niveau de la répartition phytogéographique, les espèces de liaison guinéo-congolaises-soudano-zambéziennes (GC-SZ) sont les plus nombreuses avec 130 espèces soit $46 \%$. On les rencontre généralement dans les savanes arbustives. On compte 88 taxons soit $31 \%$ des espèces dans la région Soudano-Zambézienne (SZ) localisées naturellement dans la partie septentrionale du pays. De plus, 52 espèces de la région GuinéoCongolaise(GC), soit $18 \%$ sont caractéristiques des forêts denses précisément les forêts sacrées des deux secteurs. Treize espèces soit $5 \%$ introduites ont été recensées dans la zone d'étude. Elles sont issues en majorité du domaine subsoudanais tableau IV.

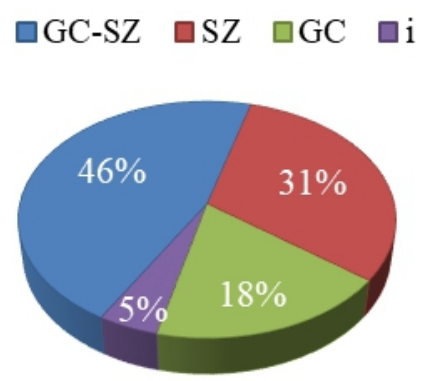

Figure 3 : Diagramme représentant la répartition phytogéographique des espèces à potentialités ornementales 
Tableau IV : Liste des espèces introduites rencontrées dans la zone d'étude

\begin{tabular}{clccc}
\hline $\mathbf{N}^{\circ}$ & \multicolumn{1}{c}{ Espèces } & Familles & Secteurs & $\begin{array}{c}\text { Type de } \\
\text { végétation * }\end{array}$ \\
\hline $\mathbf{1}$ & Bauhinia reticulata L. & Fabaceae & SubS & S \\
$\mathbf{2}$ & Bixa orellana L. & Bixaceae & SubS & J \\
$\mathbf{3}$ & Cassia surattensis Burm.f. & Fabaceae & SubS & J \\
$\mathbf{4}$ & Crescentia cujete L. & Bignoniaceae & SubS & J \\
$\mathbf{5}$ & Erythrina indica Lam. & Fabaceae & Sou & S \\
$\mathbf{6}$ & Eugenia malaccensis L. & Myrtaceae & SubS & S \\
$\mathbf{7}$ & Lagerstroemia indica Pers. & Lythraceae & SubS & S \\
$\mathbf{8}$ & Lagerstroemia speciosa Pers. & Lythraceae & SubS & S \\
$\mathbf{9}$ & Leucaena leucocephala De Wilt & Fabaceae & SubS & J \\
$\mathbf{1 0}$ & Melia azedarach L. & Meliaceae & SubS & S \\
$\mathbf{1 1}$ & Ricinus communis L. & Euphorbiaceae & Sou; SubS & S; J \\
$\mathbf{1 2}$ & Tagetes erecta L. & Asteraceae & SubS & S \\
$\mathbf{1 3}$ & Turnera ulmifolia L. & Passifloraceae & SubS & S \\
\hline
\end{tabular}

* S : Savane ; J : Jachère ; Subs : Subsoudanais ; Sou : soudanais

\section{Types décoratifs caractérisant les plantes de notre étude}

Les espèces recensées se répartissent en six groupes selon le type décoratif. Ce sont : la fleur, l'allure ou port décoratif, les feuilles, les fruits, la sève et la graine.

Parmi ces caractères de beauté, la fleur $(57,05 \%)$ est l'organe qui a permis de sélectionner plus d'espèces à potentialité décorative (Figure 4). Elle est suivie de l'allure $(21,47 \%)$ et des fruits $(12,88 \%)$.Dans $0,3 \%$ des cas, la sève peut être un critère d'ornement comme celle de Daniellia oliveri Hutch. \& Dalz. (Fabaceae) qui est utilisée pour la confection des colliers traditionnels (figure 5).

\section{Écologie des plantes recensées}

Sur le plan écologique, les plantes identifiées appartiennent à de milieux divers. On les rencontre aussi bien en milieu aquatique qu'en milieu terrestre. Les espèces comme Ceratopteris cornuta (P. Beauv.) Lepr. (Adiantaceae) et Pistia stratiotes L. (Araceae) sont des hydrophytes, d'autres espèces comme Senna podocarpa Guill. \&Perr. (Fabaceae) sont des plantes rustiques. Nous avons noté aussi la présence de certaines plantes rudérales telles que Setaria barbata (Lam.) Kunth (Poaceae). 


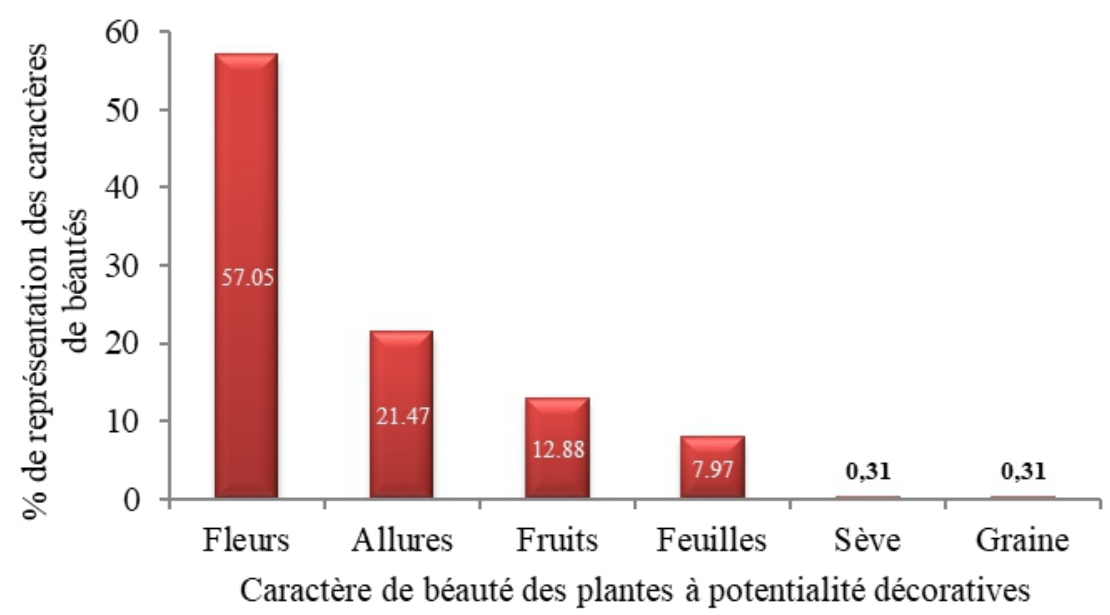

Figure 4 : Diagramme en bâton illustrant les proportions des différents caractères de beauté des plantes à potentialité décorative

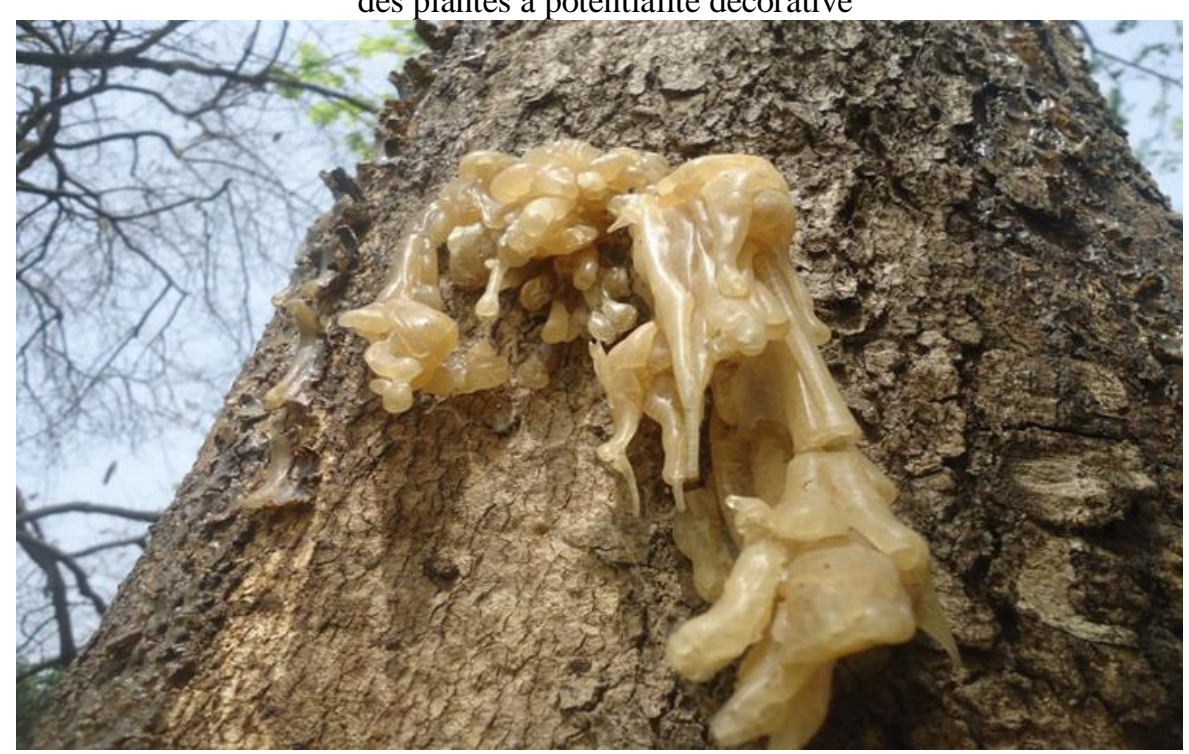

Photo : Amani, Korhogo (2017)

Figure 5 : Sève de Daniellia oliveri Hutch. \&Dalz. (Fabaceae)

\section{Diversité de la flore}

La valeur du coefficient de similitude de Sorensen pour les deux secteurs est égale à $17,03 \%$. Cette valeur est inférieure à $50 \%$, ce qui indique qu'au niveau de la flore, les formations végétales des deux secteurs sont différentes.

De plus, en ce qui concerne les indices de diversité, les sept familles présentant des indices de diversité les plus élevés sont consignés dans le tableau V. Ce sont les Fabaceae, Apocynaceae, Acanthaceae, Asteraceae, Anarcadiaceae, Combretaceae et Lamiaceae. 
Tableau V : Indices de diversité des genres et des familles

\begin{tabular}{lcccc}
\hline Famille & $\begin{array}{c}\text { Nombre } \\
\text { d'espèces }\end{array}$ & $\begin{array}{c}\text { Nombre } \\
\text { de genres }\end{array}$ & IDg & IDf \\
\hline Fabaceae & 34 & 30 & 0,104 & 0,425 \\
Apocynaceae & 31 & 17 & 0,059 & 0,388 \\
Acanthaceae & 20 & 14 & 0,048 & 0,250 \\
Asteraceae & 19 & 17 & 0,059 & 0,254 \\
Anarcadiaceae & 16 & 10 & 0,035 & 0,200 \\
Combretaceae & 14 & 8 & 0,028 & 0,167 \\
Lamiaceae & 12 & 9 & 0,031 & 0,150 \\
\hline
\end{tabular}

IDg : Indice de Diversité Générique ; IDf : Indice de diversité de Famille

\section{Les espèces à statut particulier}

Parmi les espèces inventoriées dans le domaine soudanais, dix présentent un statut particulier, soit une proportion de 3,83\% de l'ensemble des espèces inventoriées (Tableau VI). Trois d'entre elles, sont inscrites sur la liste rouge de l'UICN (2018). Il s'agit de Hygrophila senegalensis, Fimbristylis ferruginea et Dioscorea quartiniana toutes localisées dans la savane herbeuse; elles sont classées comme des espèces à risques mineurs (LC). Deux espèces vulnérables $(\mathrm{Vu})$ ont été identifiées dans le secteur subsoudanais. Ce sont Vitellaria paradoxa dans la savane naturelle arbustive et Albizia ferruginea dans la savane arborée.

Quatre taxons à risques mineurs(LC) ont été rencontrés dans des végétations différentes. Il s'agit de Tacca leontopetaloides et Commelina benghalensis localisées dans la savane herbeuse ; Gloriosa superba dans la savane arbustive et Millettia zechiana, rencontré dans un ilot de forêt dense sacrée. Deux espèces, selon Aké Assi (2001) sont signalées l'une rare, en voie d'extinction (PRE) pour Pararistolochia goldieana, une liane localisée dans le secteur subsoudanais et l'autre, Dioscorea quartiniana du secteur soudanais, devenue rare et en voie d'extinction (PDE).

Les taxons à statuts particuliers, sont en majorité des herbes et sont localisées en grande partie dans les savanes des différents secteurs étudiés. 
Tableau VI : Espèces à statut particulier

\begin{tabular}{|c|c|c|c|c|c|c|}
\hline $\mathrm{N}^{\circ}$ & Secteurs & Espèces & Familles & $\begin{array}{l}\text { Statut } \\
\text { UICN }\end{array}$ & $\begin{array}{c}\text { Statut } \\
\text { Aké } \\
\text { Assi }\end{array}$ & $\begin{array}{l}\text { Type de } \\
\text { végétation* }\end{array}$ \\
\hline 1 & \multirow{8}{*}{ Subsoudanais } & Albizia ferruginea Benth. & Fabaceae & VU & \multirow{8}{*}{ PRE } & $\mathrm{S}$ \\
\hline 2 & & Commelina benghalensis L. & Commelinaceae & $\mathrm{LC}$ & & $\mathrm{S}$ \\
\hline 3 & & Gloriosa superba $\mathrm{L}$. & Colchicaceae & $\mathrm{LC}$ & & $\mathrm{S}$ \\
\hline 4 & & Millettia zechiana Harms & Fabaceae & $\mathrm{LC}$ & & $\mathrm{F}$ \\
\hline 5 & & Pararistolochia goldieana Hook & Aristolochiaceae & & & $S$ \\
\hline 6 & & Tacca leontopetaloides O.kuntze & Dioscoreaceae & $\mathrm{LC}$ & & $\mathrm{S}$ \\
\hline 7 & & Vitellaria paradoxa C.F.Gaertn. & Sapotaceae & VU & & $\mathrm{S}$ \\
\hline 8 & & $\begin{array}{l}\text { Cyphostemma ornatum A.Chev. ex } \\
\text { Hutch. \& Dalziel }\end{array}$ & Vitaceae & GCi & & $\mathrm{J}$ \\
\hline 9 & \multirow{3}{*}{ Soudanais } & Hygrophila senegalensis Anderson & Acanthaceae & LC & & $\mathrm{S}$ \\
\hline 10 & & Fimbristylis ferruginea Vahl. & Cyperaceae & $\mathrm{LC}$ & & $S$ \\
\hline 11 & & Dioscorea quartiniana Kunth & Dioscoreaceae & $\mathrm{LC}$ & PDE & $\mathrm{S}$ \\
\hline
\end{tabular}

\section{Discussion}

Les inventaires floristiques menés dans le domaine soudanais de la Côte d'Ivoire ont permis de recenser 287 espèces à potentialité ornementale appartenant à 79 familles. Ce nombre d'espèces est supérieur à celui obtenu par Aké Assi E. (2015) avec 124 espèces. Cela s’explique par le fait que notre étude ne s'est pas déroulée dans les mêmes zones phytogéographiques que celles où Aké Assi E. (2015) a réalisé la sienne. De plus, le nombre de villes visitées dans notre étude est supérieur aux siennes. Ce nombre élevé de sites inventoriés pourrait expliquer le nombre élevé d'espèces répertoriées. Par ailleurs, ce nombre élevé (287) d'espèces recensées montre que la végétation du Nord de la Côte d'Ivoire regorge de nombreuses plantes à potentialité décorative. Ainsi, la connaissance de cette flore horticole naturelle constitue une base pour la valorisation de ces plantes à potentialité décorative et la vulgarisation de celles-ci par la domestication. Selon Melom et al. (2015), la connaissance de la flore et de la végétation d'une localité donnée est un outil indispensable pour appuyer les politiques de développement durable. Les familles les plus représentées sont les Fabaceae, Apocynaceae, Acanthaceae, Asteraceae et Anacardiaceae. Ces observations sont différentes de celles faites par Kouadio et al. (2016). En effet, les travaux de ces auteurs réalisés dans la ville d'Abidjan ont montré que les Euphorbiaceae, les Fabaceae, les Arecaceae, et les Moraceae sont les plus représentés dans cette agglomération. Cette différence est liée au fait que leur étude, s'est essentiellement focalisée sur la flore ornementale urbaine dans laquelle la sélection des espèces est fortement liée à la volonté humaine. Néanmoins, les types de végétations se rapprochent de ceux de Aké Assi E. (2015). En effet, ces deux études se sont déroulées dans le milieu naturel et ont consisté en l'inventaire de la flore ornementale indigène de la Côte d'Ivoire. La 
prédominance de la famille des Fabaceae s'expliquerait par le fait qu'elle représente, du point de vue du nombre d'espèces qu'elle contient, la famille la plus importante de la flore ivoirienne (Aké Assi 1984 ; 2001 ; 2002). Ces résultats corroborent ceux de Radji et al. (2013) qui ont également trouvé que beaucoup d'espèces ornementales appartiennent à cette famille des Fabaceae. Aussi, le nombre élevé d'espèces de Fabaceae serait-il dû au fait que les espèces de cette famille auraient une large diffusion dans divers milieux écologiques (Abdelguerfi et al., 2004). Le nombre de familles diffère d'un secteur écologique à l'autre. Cela s'explique par le fait que les espèces sont reparties en fonction du secteur écologique qui leur est favorable (Aké Assi E. et al., 2018).La dominance des microphanérophytes $(42,9 \%)$ et des herbes (45\%) concordent avec le type de végétation que l'on rencontre dans la zone d'étude. En effet, les formations arbustives (Mahamane et al., 2007) et herbeuses (Valentin et al., 2014) sont les types morphologiques les plus répandus le domaine soudanais. Par ailleurs, le nombre élevé des microphanérophytes et des herbes pourrait également s'expliquer par la variation des saisons. En effet, selon Valentin et al., (2014), la savane arbustive est relayée par une savane herbeuse dans le domaine soudanais ivoirien en saison pluvieuse. La présence d'espèces à statut particulier (endémiques, rares et menacées d'extinction de l'UICN (2018), plantes pour lesquelles les efforts de conservations doivent être prioritaires (Myers et al., 2000), témoigne que la flore ornementale naturelle du domaine soudanais possède une grande diversité. Leur faible présence indique que la végétation de la zone d'étude est fortement perturbée à cause des activités anthropiques. En effet, le Nord de la Côte d'Ivoire est sérieusement menacé, en raison de la forte régression des milieux naturels sous l'action du climat et de l'homme (feux de brousse) selon Kouadio et al. (2013). C'est pour cette raison que l'espèce Cyphostemma ornatum (Vitaceae), la seule espèce endémique à potentialité décorative recensée dans le nord ivoirien, nécessite une attention très particulière.

La prédominance des fleurs $(57,05 \%)$, comme critère de beauté a déjà été signalée par Aké Assi E. (2015). De même, l'utilisation de l'allure ou du port décoratif comme critère de sélection pour nos espèces à potentialité décorative, à également déjà servi à classifier les espèces ornementales à valeurs thérapeutiques au Togo (Radji et al., 2013).

Au plan écologique, les hydrophytes ne sont représentés que par les espèces Ceratopteris cornuta (P. Beauv) Lepr. (Adiantaceae) et Pistia stratiotes L. (Araceae). Cette rareté des hydrophytes pourrait s'expliquer la présence de retenues d'eau, qui ne serait que saisonniéres qui favorisent leur présence. 


\section{Conclusion}

$\mathrm{Au}$ terme de cette étude, 287 espèces végétales reparties entre 220 genres et 79 familles ont été inventoriées. Les familles les plus nombreuses sont les Fabaceae, Apocynaceae, Acanthaceae, Asteraceae et Anacardiaceae. La diversité des espèces à potentialité décorative se traduit également par la présence des espèces à statut particulier telles que l'espèce endémique ivoirienne (Cyphostemma ornatum) et 3 espèces endémiques Ouest africaines Culcasia Thunbergia, togoensis liberica et Senna podocarpa puis celles de la liste rouge de 1'UICN (9 espèces). La zone abrite une flore très diversifiée et une variabilité de milieu. Les formations forestières de la zone d'étude sont les plus riches et les plus diversifiées. En plus des services de régulation qu'elles apportent aux écosystèmes, les plantes à potentialité décorative jouent un rôle important dans la vie et le bien-être des populations en générale. Cette étude sur les plantes à potentialité décorative est la première réalisée dans le domaine soudanien ivoirien. D'autres contributions sont attendues en vue de compléter la liste des espèces à potentialité décorative.

\section{Remerciements}

Nous remercions le Centre National de Floristique (CNF) sis à l'Université Félix Houphouët Boigny de Côte d'Ivoire, pour les herbiers mis à notre disposition pour l'identification des espèces.

\section{References:}

1. Abdelguerfi, A. et Abdelguerfi-Laouar, M. Z. (2004). Les ressources génétiques d'intérêt fourrager et/ou pastoral : Diversité, collecte et valorisation au niveau méditerranéen Cahiers Options Méditerranéennes; n. 62 29-41 p.

2. Aké Assi, E., Kouassi, A.F., N'Goran, K.B., Yao, K., N'Guessan, K. (2018). Diversité floristique des plantes à potentialité décorative issues des formations naturelles de la flore du sud de la côte d'ivoire PASRES N³ Abidjan, Côte d'ivoire 15P.

3. Aké Assi, E. (2015). Plantes à potentialité décorative de la flore du Sud de la Côte d'Ivoire : études taxinomique, ethnobotanique et essai de domestication de Thunbergia atacorensis Akoegninou \& Lisowski (Acanthaceae), une espèce nouvellement introduite. Thèse de Doctorat, UFR Biosciences, Université Félix Houphouët-Boigny, Côte d'Ivoire $207 \mathrm{p}$.

4. Aké Assi, E., N'Guessan, K., Akédrin, T.N. (2010). Atlas de la Biodiversité de l'Afrique de l'Ouest, Côte d'ivoire, Tome III, P324325 . 
5. Aké Assi, L. (1984). Flore de la Côte d'Ivoire. Étude descriptive et biogéographique avec quelques notes ethnobotaniques. Tome. II. III. Thèse de Doctorat, Université d'Abidjan, 1205p.

6. Aké Assi, L. (2001). Flore de la Côte d'Ivoire 1, catalogue, systématique, biogéographie et Écologie. Genève, Suisse : Conservatoire et jardin Botanique de Genève ; Boissiera 57, 396 p.

7. Aké Assi, L. (2002). Flore de la Côte d'Ivoire 2, catalogue, systématique, biogéographie et Écologie. Genève, Suisse Conservatoire et Jardin Botanique de Genève, Boisseria 58, 441 p.

8. Ambé, GA. (2001). Les fruits sauvages comestibles des savanes guinéennes de Côte-d'Ivoire: état de la connaissance par une population locale, les Malinké. Biotechnologie, agronomie, société, et environnement 5(1): 43-58.

9. APG VI (Angiosperm Phylogeny Group) (2016). Classification of the orders and families of flowering plants. Botanical Journal of the Linnean Society, 31 (2): 71-80.

10. Bamba, I., Barima, Y.S.S. Bogaert J. (2010). Influence de la densité de la population sur la structure spatiale d'un paysage forestier dans le bassin du Congo en R. D. Congo, Tropical Conservation Science vol. 3 (1) : $31-44$.

11. Barloy, J. (1984). Biologie de l'élaboration du rendement. in: Physiologie du Mai's (A. Gallaised.) INRA, pp. 163-180.

12. Bertault, J. (1986). Étude de l'effet d'interventions sylvicoles sur la régénération naturelle au sein d'un périmètre expérimental d'aménagement en forêt dense humide de Côte d'Ivoire. Thèse de l'Université de Nancy, 254 p.

13. BNETD, F. (2015). Identification, analyse et cartographie des causes de la déforestation et de la dégradation des forêts en Côte d'Ivoire.

14. Devineau JL., Charles L., Roger V. (1984).Évolution de la diversité spécifique du peuplement ligneux dans une succession préforestière de colonisation d'une savane protégée des feux (Lamto, Côte d'Ivoire). Candollea, pp.103-134.

15. Djato, K.K. (2001). Crédit agricole et efficacité de la production agricole en Côte d'Ivoire. Économie rurale, 263(1): 92-104.

16. Ehui, S.K., Hertel, T.W. (1989). Deforestation and agricultural productivity in the Côte d'Ivoire. American Journal of Agricultural Economics, pp 703-711.

17. Guillaumet J. L. (1967). Recherches sur la végétation et la flore de la région du Bas-Cavally (Côte d'Ivoire). Mémoires ORSTOM n ${ }^{\circ} 20$, Paris, $247 \mathrm{p}$.

18. Guillaumet, J.L., Adjanohoun, E. (1971)."La végétation de la Côte d'Ivoire." pp: 161-263 
19. Kouadio, K.B., N’Da, D.H., Vroh, Bi T.A., Zobi, I.C., N'Guessan, K.E. (2013). Dynamique de la végétation et fréquence des feux de brousse dans la réserve de faune d'Abokouamekro (centre, Côte d'Ivoire). European Scientific Journal 9 (35) pp : 179-192

20. Kouadio, Y. J. C., Vroh, B. T. A., Bi, Z. B. G., Yao, C. Y. A., N'Guessan, K. E. (2016). Évaluation de la diversité et estimation de la biomasse des arbres d'alignement des communes du Plateau et de Cocody (Abidjan-Côte d'Ivoire). Journal of Applied Biosciences, 97, 9141-9151.

21. Kouamé, N. F. (1998). Influence de l'exploitation forestière sur la végétation et la flore de les forêts classée du Haut-Sassandra (CentreOuest de la Côte d'Ivoire). Thèse Doctorat 3è Cycle, UFR Biosciences, Université Cocody- Abidjan, Côte d'Ivoire, 227 p.

22. Mahamane, A., Mahamane, S., Lejoly, J. (2007). Phénologie de quelques espèces ligneuses $\mathrm{du}$ parc national $\mathrm{du} \ll \mathrm{W} » \mathrm{du}$ Niger. Sécheresse, 18(4), 354-8.

23. Malan, D F., Laurent, A A., Fézan,H.T.B., Danho, N.F.R. (2007). Diversité floristique du parc national des îles Ehotilé (littoral est de la Côte d'Ivoire) $\mathrm{N}^{\circ} 292$ (2) Diversité Biologique 49 Flore, Côte D'ivoire $10 \mathrm{p}$.

24. Mboh, H., (2001). Vegetation assessment of Takamanda forest reserve and comparism with Campo Ma'an and Ejagham forest reserves: Thesis, Faculty of Agronomy and Agricultural Sciences, University of Dschang. 112p.

25. Melom, S., Mbayngone, E., Bechir, AB., Ratnan,N., Mapongmetsem, M. (2015). Caractéristiques floristique et écologique des formations végétales de Massenya au Tchad (Afrique centrale). Journal of Animal \& Plant Sciences, 25(1) : 3799-3813.

26. Myers, N., Mittermeier, R. A., Mittermeier, C. G., Fonseca, Da., G. A., Kent, J. (2000). Biodiversity hotspots for conservation priorities. Nature, 403(6772), 853.

27. Radji, R., Kokou, K., Akpagana, K. (2010). Étude diagnostique de la flore ornementale togolaise Laboratoire de Botanique et Ecologie Végétale, Faculté des Sciences, Université de Lomé, BP : 1515, Lomé, TOGO. Journal of Biological and Chemical Sciences 4(2): 491-508, 1991-8631 17p.

28. Radji, R., Kokou, K., Bandje, A., Issifou, L., Edorh, T. (2013). Diversité et dynamique des assemblages phytoplanctoniques dans les écosystèmes aquatiques au Sud du Togo Laboratoire de Botanique et Ecologie Végétale. Université de Lomé,Togo Afrique Science 11p. 
29. Radji, R., Kokou, K. (2013.) Classification et valeurs thérapeutiques des plantes ornementales du Togo. [VertigO] La revue électronique en sciences de l'environnement, vol. 13, no 3.

30. Raunkier, C. (1934). The life forms of plants and statistical plant of geography, Oxford Londres, Angleterre, 632 p.

31. Sangne, YC. (2009). Dynamique du couvert forestier d'une aire protégée soumise aux pressions anthropiques : cas de la Forêt Classée de Téné dans le Département d'Oumé (Centre-ouest de la Côte d'Ivoire). Thèse de Doctorat, Université de Cocody-Abidjan.

32. SODEFOR (1996). Plan d'aménagement de la forêt classée de Bouaflé. Ministère de l'agriculture et des ressources animales, pp 361

33. Sorensen, T. (1948). A method of establishing groups of equal amplitude in plant sociology based on similarity of species content ; Det Kongelige Danske Videskabernes Selskab, Biologiskes kriffer, 5(4): 1-34.

34. Traoré, L, Ouédraogo, I, Ouédraogo, A., Thiombiano, A. (2011). Perceptions, usages et vulnérabilité des ressources végétales ligneuses dans le Sud-Ouest du Burkina Faso. International Journal of Biological and Chemical Sciences, 5(1).pp 34-56.

35. UICN, (2018). Red List of Threatened Species. http://www.iucnredlist. org/. (consulté le 20 février 2018)

36. Valentin, C., Janeau J.L. (2014). Les risques de dégradation structurale de la surface des sols en savane humide (Côte d'ivoire) Laboratoire de Pédologie expérimentale, ORSTOM, BP V-51, Abidjan, Côte d'Ivoire.23p.

37. Vroh, BTA. (2013). Évaluation de la dynamique de la végétation dans les zones agricoles d'Azaguié (Sud-est Côte d'Ivoire). Thèse de Doctorat unique, UFR Biosciences, Université Félix HouphouëtBoigny, Abidjan, Côte d'Ivoire, 163 p.

38. Wezel, A., Haigis, J. (2000). Farmer's perception of vegetation changes in semi-arid Niger, 523-534.

39. Wezel, A., Lykke, AM. (2006). Woody vegetation in Sahelian West Africa: evidence from local knowledge. Environ Dev Sustain, 8: 553567.

40. White, F. (1983). The Vegetation of Africa: A descriptive memoir to accompany UNESCO/AETFAT/UNSO vegetation maps of Africa. Paris.

41. Yao, A. (2013). Diversité floristique et valeur de la forêt sacrée Bokasso (Est de la Côte d'Ivoire) pour la conservation. VertigO-la revue électronique en sciences de l'environnement, 13(1). 